\title{
Development of a Landing Mechanism for Asteroids with Soft Surface
}

\author{
Zhijun Zhao, Jingdong Zhao, and Hong Liu \\ State Key Laboratory of Robotics and System, Harbin Institute of Technology, Harbin 150080, China \\ Correspondence should be addressed to Jingdong Zhao; zhaozhj@yahoo.com
}

Received 2 July 2013; Revised 5 September 2013; Accepted 15 September 2013

Academic Editor: Mahmut Reyhanoglu

Copyright (C) 2013 Zhijun Zhao et al. This is an open access article distributed under the Creative Commons Attribution License, which permits unrestricted use, distribution, and reproduction in any medium, provided the original work is properly cited.

A landing mechanism to an asteroid with soft surface is developed. It consists of three landing feet, landing legs, cardan element, damping element, equipment base, anchoring system, and so on. Static structural analysis and modal analysis are carried out to check the strength and natural frequency of the landing mechanism with FEA. Testing platform for the anchoring system is introduced, and then the penetrating and anchoring tests of the anchoring system are carried out in different media. It shows that cohesion of the media has large influence on the penetrating and anchoring performance of the anchoring system. Landing tests of the landing mechanism with different velocities under simulated microgravity environment are carried out on the air-floating platform, and the impact accelerations are measured by the sensors on the landing mechanism. At the same time, these impact accelerations are processed by spectrum analysis to find the natural frequency of the landing mechanism.

\section{Introduction}

Asteroids originate from the early formation of the solar system and are distributed almost everywhere of the universe. They are similar to the comets in size, mass, orbital character, formation time, and so forth. Some asteroids are rich in minerals and raw materials of the solar system's initial formation, so they have very important scientific and economic values $[1,2]$. Currently, landing on the small bodies, including asteroids and comets, is a hot spot to explore. So far, Japan has already landed and sampled successfully from an asteroid [3]. Besides, NASA has devoted lots of energy in small body landing explorations, such as NEAR spacecraft $[4,5]$. At the same time, ESA has launched the Rosetta lander to explore a comet by landing on it [6]. Asteroids and comets are similar in some physical properties constraining the design of the lander as mentioned above; thus some design experiences of the comet lander are significant in guiding the design of the asteroid lander. Therefore, Rosetta lander is presented even though it is a comet lander.

There are many approaches to explore the asteroids, such as observing on the ground, detecting by the orbital spacecraft [7], sampling return $[8,9]$, and exploring by landing on them $[6,10]$. Among them, landing exploration is an efficient way to explore the asteroid. The Moon and Mars have been explored successfully by landing on them. The merits of the landing exploration are as follows: (1) landing exploration could analyze the asteroid's media in situ; (2) landing exploration can get comprehensive data of the asteroid, and these data are very useful to future asteroid explorations, such as changing the orbits of the asteroids to avoid them striking the earth [11] or guiding the asteroid rich in mineral, valuable metal, water, and so forth [12] to land on the earth to solve the energy shortage problem and at the same time to gain great economic value. Moreover, we can select an asteroid to be the base to explore the solar system [11] and so on.

Thus, it is necessary to develop the landing mechanism for landing on the asteroid. Up to now, landing mechanisms have been applied to Moon lander, Mars lander, and comet lander. NASA has developed lots of landing mechanisms with three or four legs for landing on the Moon and Mars, and their legs are foldable or not. Most of them are cushioned by honeycomb aluminum $[13,14]$. DLR and MPS also have developed the comet landing mechanism for ESA [15]. It has three legs, cushioned by electromagnetic damping, and it can adjust the attitude of the equipment base. The most special characteristic of the Rosetta landing mechanism is that it has an anchoring mechanism to avoid the lander flowing away 
TABLE 1: Landing environments of some asteroids and comets.

\begin{tabular}{|c|c|c|c|c|c|}
\hline & Eros (asteroid) $[1,18]$ & Itokawa (asteroid) [19] & Lutetia (asteroid) [20] & $\begin{array}{c}\text { Churyumov- } \\
\text { Gerasimenko (comet) } \\
{[21]}\end{array}$ & $\begin{array}{c}\text { Wirtanen (comet) } \\
{[16,22]}\end{array}$ \\
\hline Diameter & $8.7-31.8 \mathrm{~km}$ & $1070 \mathrm{~m} \times 588 \mathrm{~m} \times 418 \mathrm{~m}$ & $98.3 \pm 5.9 \mathrm{~km}$ & $3.44 \mathrm{~km}$ & $1.2 \mathrm{~km}$ \\
\hline Gravity & $0.0024-0.0059 \mathrm{~m} / \mathrm{s}^{2}$ & $\begin{array}{c}10^{-6}-10^{-5} \mathrm{~m} / \mathrm{s}^{2} \\
\left(m=3.51 \times 10^{10} \mathrm{~kg}\right)\end{array}$ & $\begin{array}{c}0.034 \mathrm{~m} / \mathrm{s}^{2} \\
\left(m=1.22 \times 10^{18} \mathrm{~kg}\right)\end{array}$ & $\begin{array}{c}10^{-4} \mathrm{~m} / \mathrm{s}^{2} \\
\left(m=8 \times 10^{12} \mathrm{~kg}\right)\end{array}$ & $10^{-4}-10^{-5} \mathrm{~m} / \mathrm{s}^{2}$ \\
\hline Escape velocity & About $10 \mathrm{~m} / \mathrm{s}$ & $2.1 \times 10^{-4} \times 5.4 \times 10^{-3} \mathrm{~m} / \mathrm{s}$ & $41 \mathrm{~m} / \mathrm{s}$ & $0.4 \mathrm{~m} / \mathrm{s}$ & $0.08-0.25 \mathrm{~m} / \mathrm{s}$ \\
\hline $\begin{array}{l}\text { Rotation } \\
\text { period }\end{array}$ & 5.27 hours & $12.1324 \pm 0.0001$ hours & $8.17 \pm 0.01$ hours & 12.4-12.7 hours & $6 \pm 0.3$ hours \\
\hline Pressure & About $0 \mathrm{~Pa}$ & About $0 \mathrm{~Pa}$ & About $0 \mathrm{~Pa}$ & About $0 \mathrm{~Pa}$ & About $0 \mathrm{~Pa}$ \\
\hline Topography & Rough & Rough & Rough & Rough & Rough \\
\hline
\end{tabular}

from the comet surface. As asteroid landing mechanism is very significant for landing on the asteroid, Asteroid Landing and In Situ Exploring (ALISE) landing mechanism to asteroid with soft surface is developed.

In the paper, landing environment of the asteroid is analyzed firstly. Then, ALISE landing mechanism is designed to suit the landing environment of the asteroid. Thirdly, finite element analysis (FEA) is used to estimate the strength and modal frequency of the landing mechanism. Fourthly, experiments to test the performance of the landing mechanism are carried out. Experiments include penetrating/anchoring tests of the anchoring system and landing tests under simulated microgravity environment generated by air-floating platform. By the way, validity of the FEA is testified by spectrum analysis of the acceleration signals on the landing mechanism when landing.

\section{Structural Design of the ALISE Landing Mechanism}

2.1. Landing Environment of the Asteroid. Landing environment is a key factor that must be considered in design of the landing mechanism. Asteroid's landing environment is different from that of the Moon and Mars but similar in some respect to that of the comet, and they are summarized in Table 1. However, surface strength of the asteroid and comet is not justified scientifically; thus there is no comparison of them in Table 1. It can be found that the gravity of the asteroid is small, rotation period is short, the surface is rough, and so on. These characteristics constrain the structural configuration of the asteroid landing mechanism and induce that it is different from that of the Moon and Mars. Some constraints in design of the asteroid landing mechanism are as follows. (1) Landing mechanism is easy to rebound and drift away because of the microgravity and large centrifugal force of the asteroid. (2) Landing mechanism will be inclined to overturn when landing because of the rough surface of the asteroid. (3) The attitude of the equipment base in the landing mechanism should be adjustable to suit the rough surface of the asteroid. (4) Soft landing is needed to avoid crashing instruments.

Besides, surface strength of the asteroid is largely different according to the compositive materials of them. Somewhere
TABLE 2: Mechanical and landing performance parameters.

\begin{tabular}{lc}
\hline Items & Values \\
\hline Mass of landing mechanism & $45 \mathrm{~kg}$ \\
Mass of payload & $\leq 55 \mathrm{~kg}$ \\
Mass of anchoring system & $1 \mathrm{~kg}$ \\
Horizontal velocity & $0.5 \mathrm{~m} / \mathrm{s} \leq V_{x} \leq 0.5 \mathrm{~m} / \mathrm{s}$ \\
Vertical velocity & $V_{y} \leq 1.5 \mathrm{~m} / \mathrm{s}$ \\
Landing slope & $\theta \leq 30^{\circ}$ \\
Tensile strength of the media & $0.5 \mathrm{MPa} \leq \tau \leq 5 \mathrm{MPa}$ \\
Anchoring time & $\leq 5 \mathrm{~s}$ \\
Anchoring force & $\geq 100 \mathrm{~N}$ \\
Penetrating velocity & $20 \mathrm{~m} / \mathrm{s} \sim 100 \mathrm{~m} / \mathrm{s}$ \\
Rewinding force & About $20 \mathrm{~N}$ \\
Thread length & About $2 \mathrm{~m}$ \\
\hline
\end{tabular}

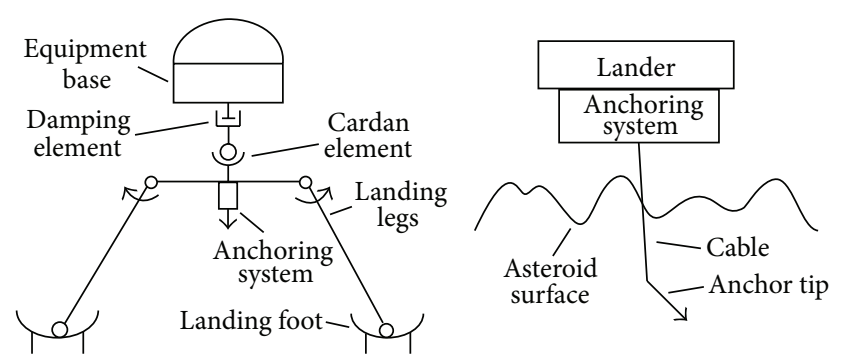

FIGURE 1: Schematic of the ALISE landing mechanism and its anchoring system.

the strength is very high; however, somewhere the strength is very low. Thus, surface strength of the asteroid also restricts the design of the landing mechanism. In the paper, ALISE landing mechanism is designed for the asteroid with soft surface.

2.2. Structural Design. The ALISE landing mechanism contains a three-leg landing gear and an anchoring system, and its schematics are shown in Figure 1. This design is inspired by the Rosetta lander and the ST4/Champollion lander $[10,16,17]$. The pictures of the landing mechanism are shown in Figure 2. The mechanical performance and landing performance parameters are shown in Table 2. 


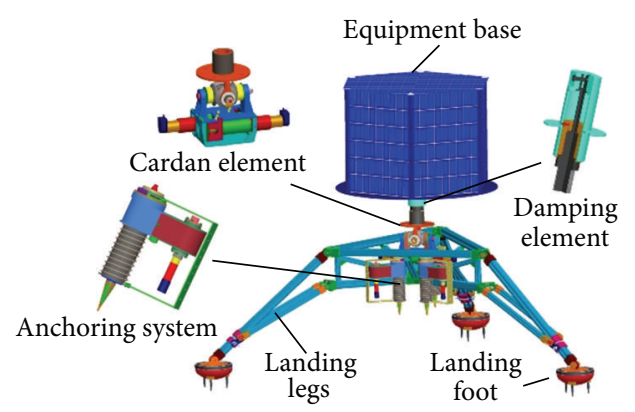

(a)

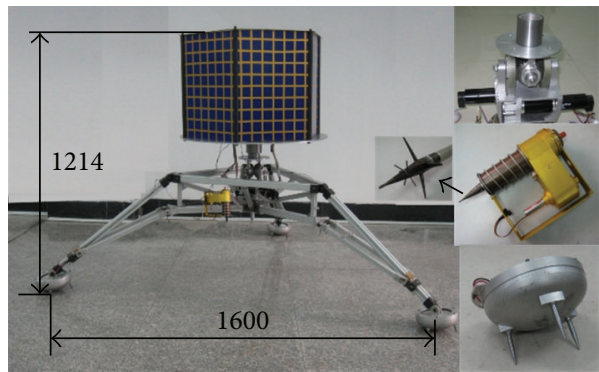

(b)

FIGURE 2: Pictures of the landing mechanism.

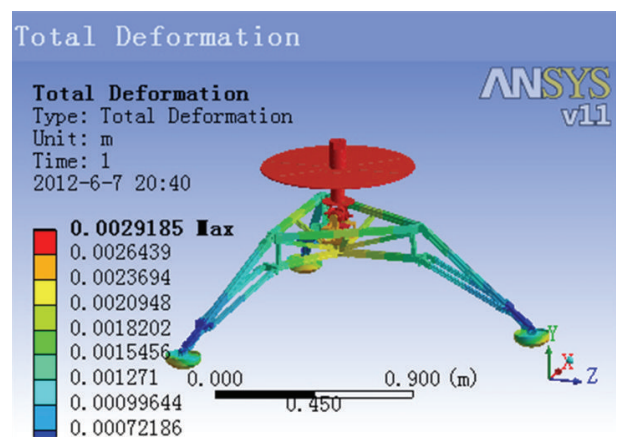

(a)

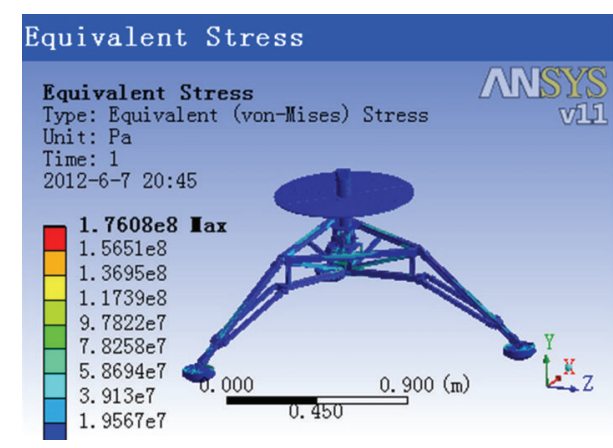

(b)

FIgURE 3: Static structural analysis of the landing mechanism by FEA.

The landing gear contains landing foot, landing leg, cardan element, damping element, equipment base, and so on. The anchoring system is used to avoid the lander flowing away under microgravity. It contains anchoring element, propulsion element, rewinding element, cushion element, and so on. The tip of the anchoring system is conical, and it could be splayed after penetrating to enhance the anchoring force.

The landing legs are foldable. There are awls beneath the landing feet to stop the landing mechanism from sliding when landing. Inside the landing feet there are contact switches generating landing signals. The cardan element could absorb the horizontal impact when landing and adjust the attitude of the equipment base after landing. The damping element working in electromagnetic principle absorbs the vertical impact when landing. Its damping constant is $900 \mathrm{Nm} / \mathrm{s}$ and stroke is about $0.13 \mathrm{~m}$. The propulsion element of the anchoring system pushes the anchoring element to penetrate into the surface of the asteroid in a high velocity. The anchoring element connects to the rewinding element with a thread and could splay after penetrating. At the time of the anchoring element penetration, the rewinding element would rewind the thread quickly. When the thread is tense instantaneously, the cushion element comprised mainly of a compression spring will absorb the impact to protect the rewinding motor. A retrorocket as a part of the control system is designed on the top of the equipment base. The retrorocket would be activated at the time of landing and supply a constant force lasting about 5 seconds toward the equipment base to prevent the landing mechanism from rebounding.

\subsection{Finite Element Analysis of the Structure}

2.3.1. Static Structural Analysis. Finite element analysis is processed to check the structural strength of the landing mechanism when landing. The allowable overloading acceleration of the equipment base, which has a mass of $78 \mathrm{~kg}$ (the total mass of the landing mechanism and payload is $100 \mathrm{~kg}$, and the total mass of landing gear, landing feet, and anchoring system is about $22 \mathrm{~kg}$ ), is set to $10 \mathrm{~g}$. Thus, the maximum inertial force acts on the landing mechanism when landing is about $7800 \mathrm{~N}$. So the force used to analyze the structural strength is $7800 \mathrm{~N}$, and the force is acting on the damping element. Besides, a planar constraint is placed on three feet. The analysis results are shown in Figure 3. The struts are manufactured from profiled aluminum. Their section is square, and the thickness is about $2.2 \mathrm{~mm}$. It can be found that the maximum deformation is about $0.0029 \mathrm{~m}$, and the maximum equivalent stress is about $176 \mathrm{MPa}$ which is less than the yield strength of the aluminum alloy 7A09 (400 $\mathrm{MPa}$ ) to manufacture the landing mechanism. So the structural strength and deformation of the landing mechanism are reasonable.

2.3.2. Modal Analysis. Modal analysis is used to understand the vibrating characters of the landing mechanism. In the 

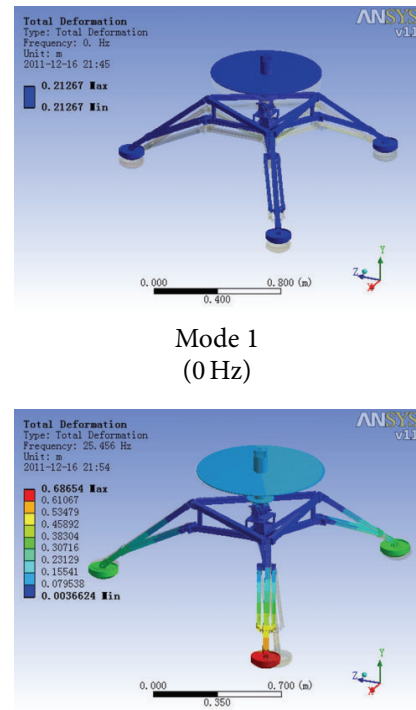

Mode 5

$(25.456 \mathrm{~Hz})$

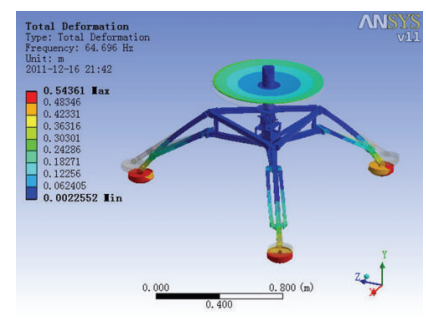

Mode 9

$(64.696 \mathrm{~Hz})$

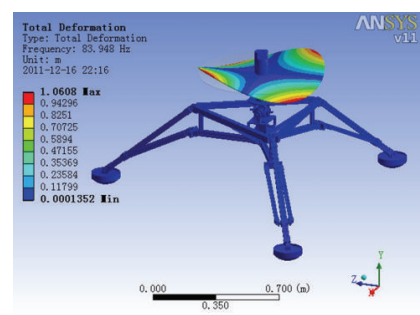

Mode 13

$(83.948 \mathrm{~Hz})$

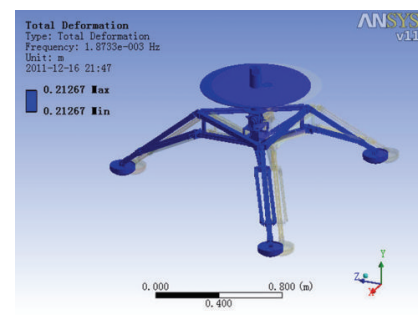

Mode 2

$(0.0019 \mathrm{~Hz})$

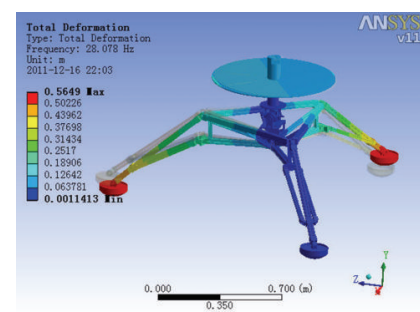

Mode 6

$(28.078 \mathrm{~Hz})$

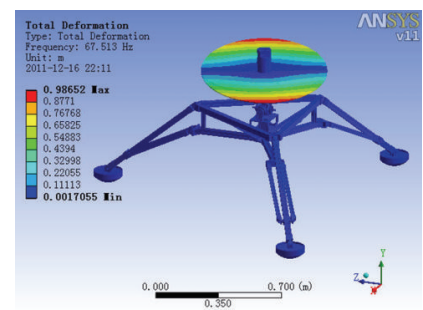

Mode 10

$(67.513 \mathrm{~Hz})$

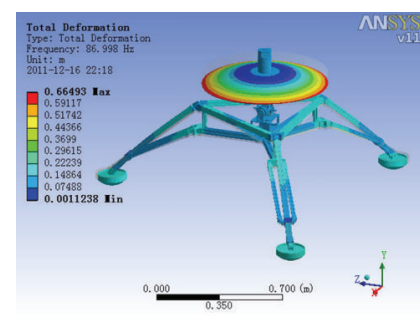

Mode 14

$(86.998 \mathrm{~Hz})$

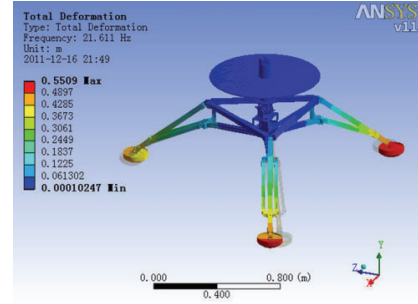

Mode 3

(21.611 Hz)

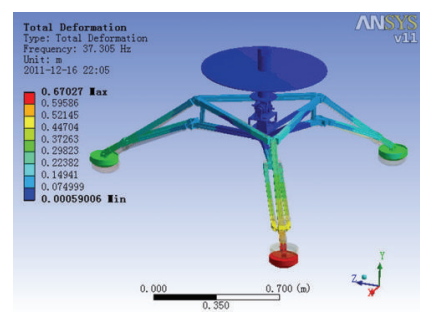

Mode 7

(37.305 Hz)

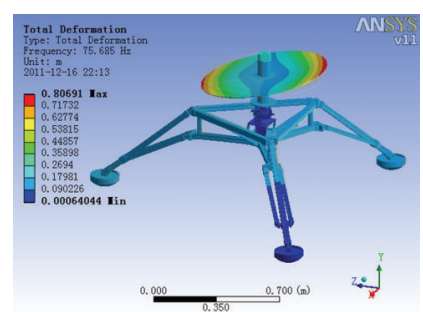

Mode 11

$(75.685 \mathrm{~Hz})$

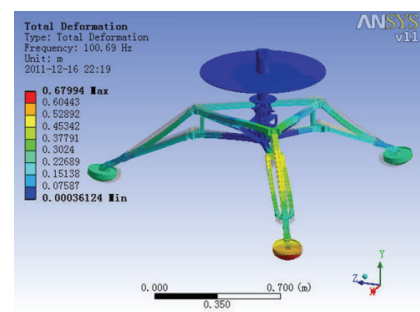

Mode 15

$(100.69 \mathrm{~Hz})$

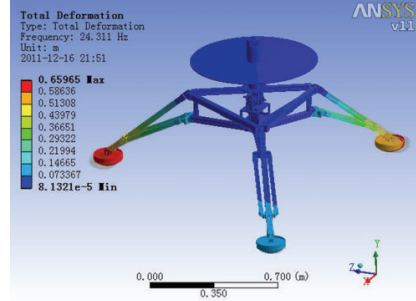

Mode 4

(24.311 Hz)

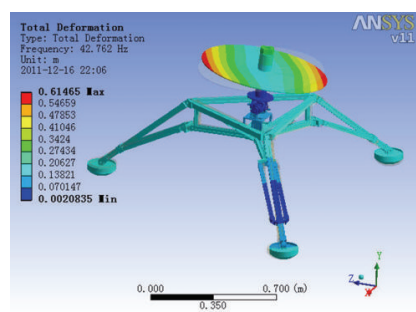

Mode 8

$(42.762 \mathrm{~Hz})$

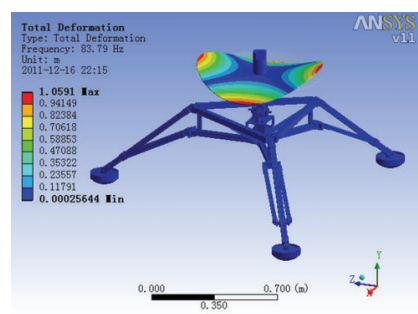

Mode 12

(83.79 Hz)

FIGURE 4: Modal analysis of the deployed landing mechanism.

paper, only the modals of the deployed states are analyzed, and these modal frequencies will be used in the experiments analyzing of landing. In modal analysis, a frictionless support constraint is placed on the landing mechanism, which simulates the constraints supporting the landing mechanism on the air-floating platform as shown in Section 3.2. The modal frequencies of the deployed landing mechanism are shown in Figure 4.

It can be found that mode 6 , mode 7 , mode 9 , and mode 15 induce the vibration of landing legs along the direction of landing ( $Y$-axis), mode 9 induces the vibration of equipment base along the direction of landing ( $Y$-axis), and modes 8,10 , and 11 induce the oscillation of the equipment base.

\section{Experiments}

\subsection{Anchoring System Tests}

3.1.1. Testing Platform. Testing platform of the anchoring system is shown in Figure 5. The anchoring element is fired by the powder which is triggered via the trigger, and the trigger is controlled by the electric cable. When the anchor body is fired by the anchoring element, the firing speed of the anchor body is measured by the speedometer, and the penetrating depth is measured by the thread on the rear of the anchor body. Then the anchor body is pulled out of the medium by the rocker-arm via the pulley. There is a force gauge between 


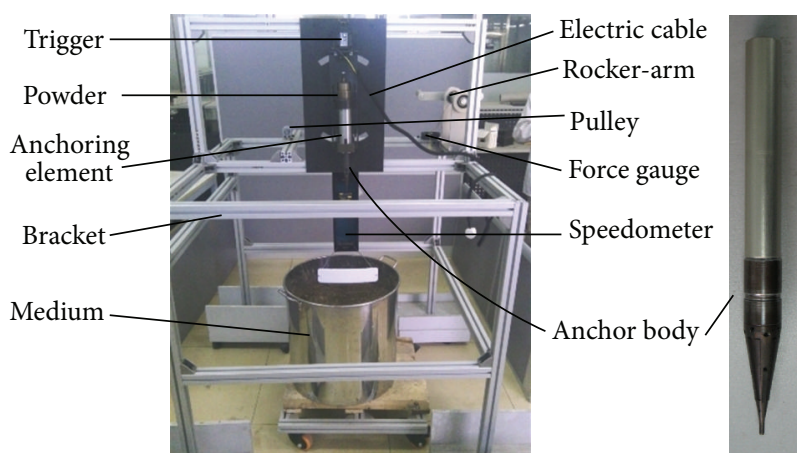

FIGURE 5: Testing platform of the anchoring system.

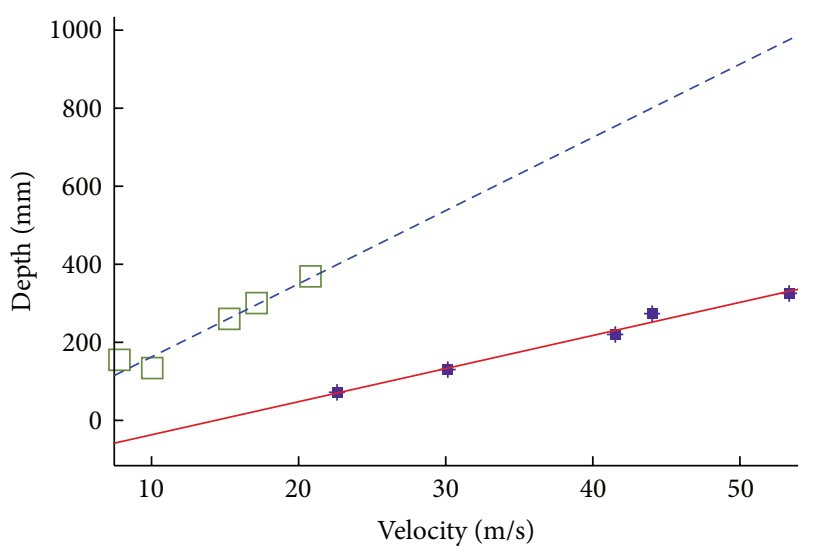

- Soil depth versus velocity Soil fitting

$$
\square \quad \text { Sand depth versus velocity }
$$$$
\text { -- - Sand fitting }
$$

FIgURE 6: Penetrating depth versus velocity in soil and sand.

the rocker-arm and the pulley; thus the anchoring force of the anchoring element will be measured. The height of the bracket could be heightened according to the thickness of the medium when the penetrating speed is high. The anchor body installed in the anchoring element is shown in Figure 5. The length of it is about $288 \mathrm{~mm}$, the diameter is about $26 \mathrm{~mm}$, and the mass is about $600 \mathrm{~g}$.

In the paper, two media (clay soil and silver sand) are used to test the performance of the anchoring system. The properties of the media are shown in Table 3. Clay soil is some cohesive and the silver is some flowing, and they represent two different types of media.

3.1.2. Testing Results and Analyses. Penetrating depth and anchoring force of the anchor body with different penetrating velocities in two media are tested.

Figure 6 shows the relationship between penetrating depth and penetrating velocity in soil and sand media, and the testing data are fitted linearly. Some conclusions could be gotten as follows: (1) cohesion of the media has a great influence of the penetrating depth; (2) penetrating depth increases almost linearly along the penetrating velocity; (3) the smaller the cohesion is, the steeper the penetrating depth changes with the penetrating velocity.

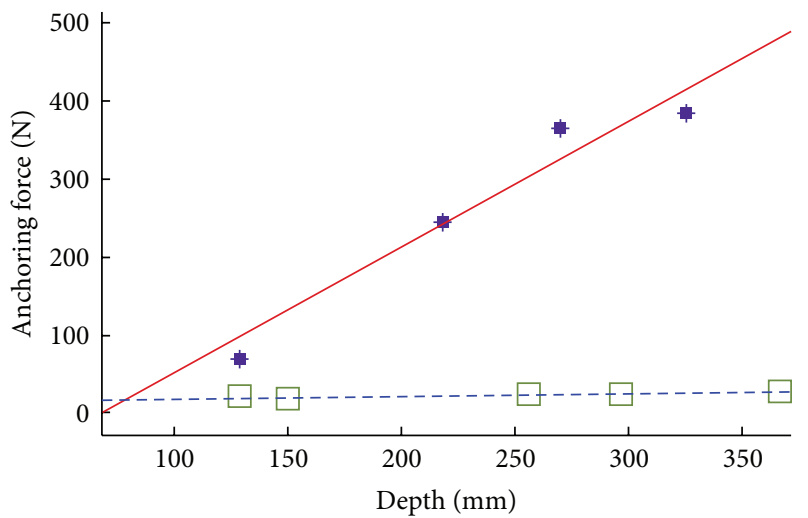

* Soil anchoring versus depth

$\square \quad$ Sand anchoring versus depth - - - Sand anchoring fitting

Figure 7: Anchoring force versus depth in soil and sand.

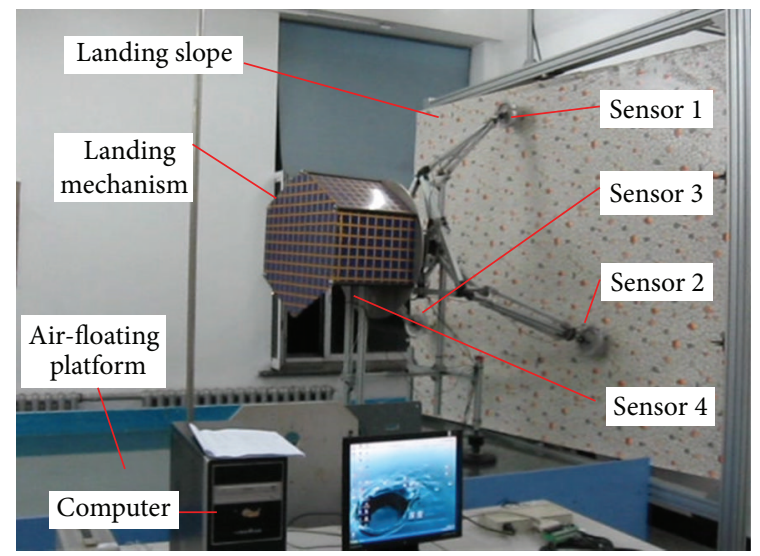

FIGURE 8: Landing mechanism on the air-floating platform.

Figure 7 shows the relationship between anchoring force and penetrating depth in soil and sand media, and the testing data are fitted linearly. Some conclusions could be gotten as follows. (1) The anchoring force increases with the depth in soil but maintains almost constant in sand. It shows that depth has less influence of the anchoring force in very low cohesion media. (2) The anchoring force in soil seems to change slowly when the depth reaches a certain value according to the testing data without fitting. However, there are only 5 groups of testing data, and this trend needs to be further verified by more testing data.

\subsection{Landing Tests under Simulated Microgravity Environment}

3.2.1. Testing Platform. The landing performance of the landing mechanism is tested under simulated microgravity environment generated on the air-floating platform. The schematic of the landing mechanism on the air-floating platform is shown in Figure 8.

Landing tests are carried out on a $10^{\circ}$ landing slope with the landing velocity increasing gradually. The landing accelerations are measured by acceleration sensors. The 
TABLE 3: Properties of the penetrating and anchoring media.

\begin{tabular}{lccccc}
\hline Media & Density & Water content & Void ratio & Cohesion & Internal frictional angle \\
\hline Clay soil & $1.89 \mathrm{~g} / \mathrm{cm}^{3}$ & $19.91 \%$ & 0.709 & $270 \mathrm{KPa}$ & $14^{\circ}$ \\
Silver sand & $1.71 \mathrm{~g} / \mathrm{cm}^{3}$ & 0 & 0.585 & $0 \mathrm{KPa}$ & $37^{\circ}$ \\
\hline
\end{tabular}

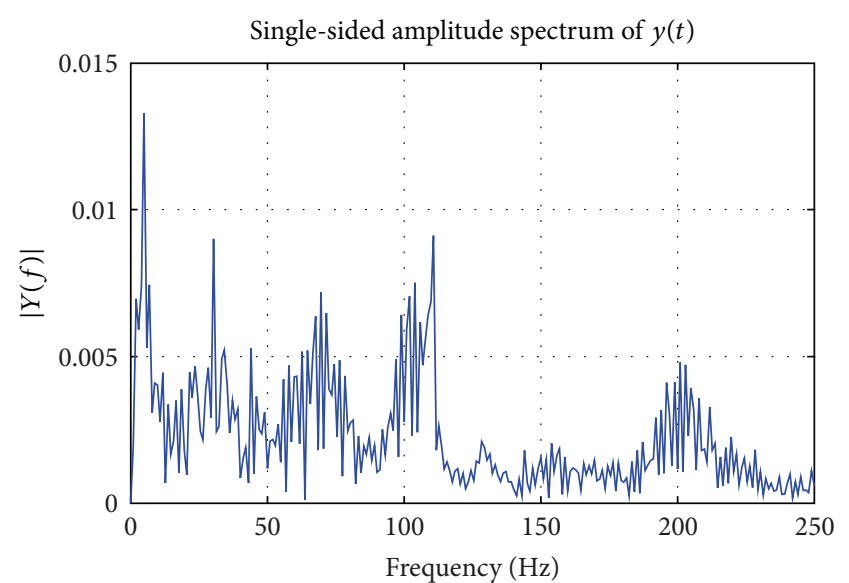

(a) Sensor 1 spectrum

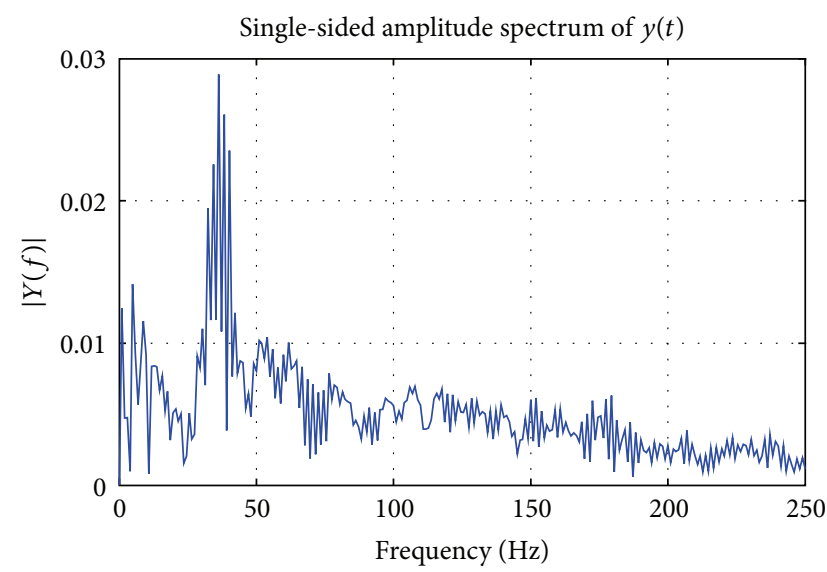

(c) Sensor 3 spectrum

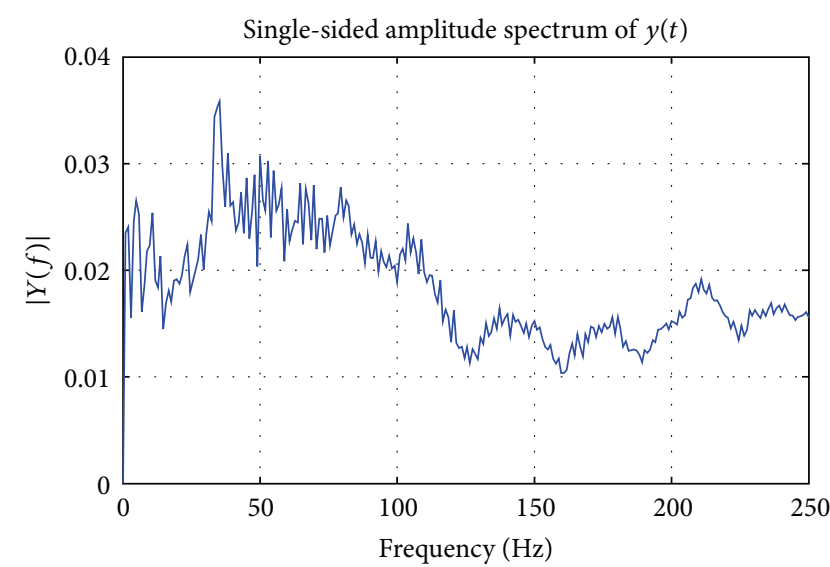

(b) Sensor 2 spectrum

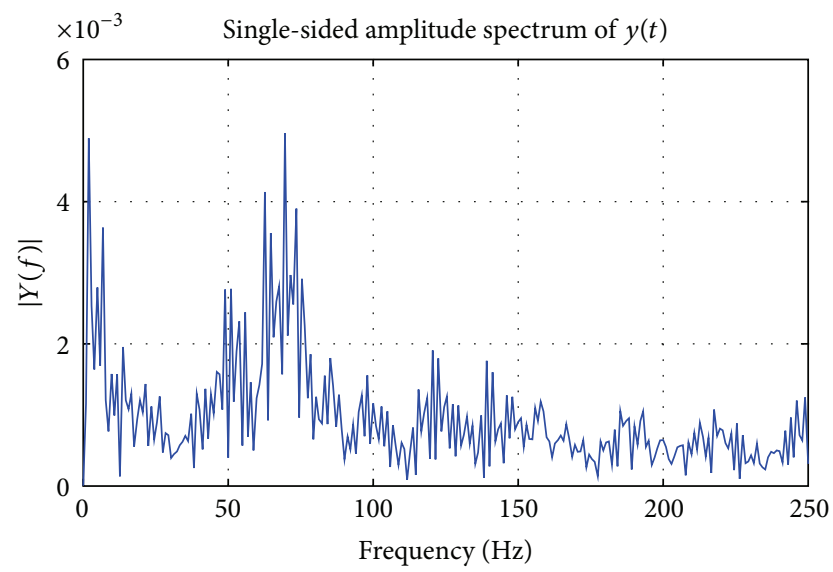

(d) Sensor 4 spectrum

FIGURE 9: Sensors spectrum of the landing mechanism with $V_{y}=0.41 \mathrm{~m} / \mathrm{s}, V_{x}=0 \mathrm{~m} / \mathrm{s}$, and $\theta=10^{\circ}$.

landing attitude of the landing mechanism and the location of the sensors are shown in Figure 8. Sensors 1, 2, and 3 located separately on three landing feet are used to measure impacting accelerations of the feet in landing direction. The indexes of the sensors are the same as those of the feet. Sensor 4 locates on the equipment base and is used to measure the acceleration of the equipment base in landing direction.

3.2.2. Testing Results and Analyses. Three groups of landing in different landing velocities are tested. The landing accelerations are filtered by a low-pass filter whose cut-off frequency is defined both by the spectrum analysis of the signals and the modal analysis of the landing mechanism in Section 2.3. The acceleration signals' spectrum analyses in different landing velocities are shown in Figures 9, 10, and 11, respectively, and the corresponding results of the acceleration signals after filtering are shown in Figures 12, 13, and 14, respectively. Besides, in three groups of landing tests, anchoring system could firmly anchor the landing mechanism to the landing slope.

The analyses of the landing tests according to Figures 9, $10,11,12,13$, and 14 are as follows.

(i) The natural frequencies of the landing mechanism obtained by spectrum analysis of the acceleration signals are basically in accordance with the results obtained by the modal analysis as shown in Table 4 . The effectiveness of the modal analysis and the landing accelerations are validated mutually.

(ii) When landing with the attitude (1-1-1 landing mode) shown in Figure 8, it can be found in Figures 12, 13 , and 14 that the landing mechanism contacts the 


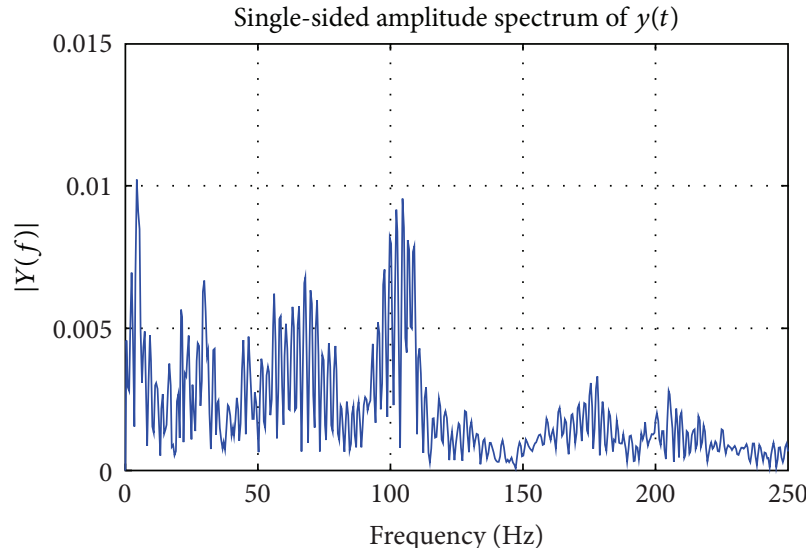

(a) Sensor 1 spectrum

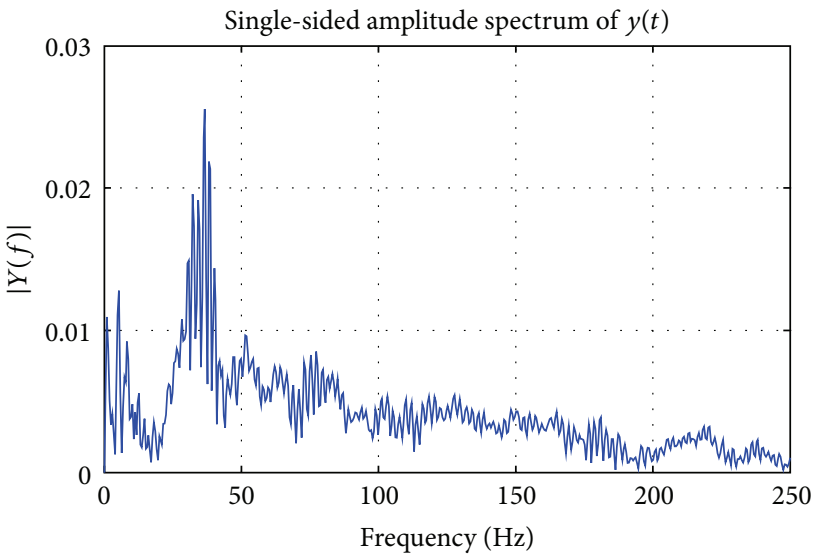

(c) Sensor 3 spectrum

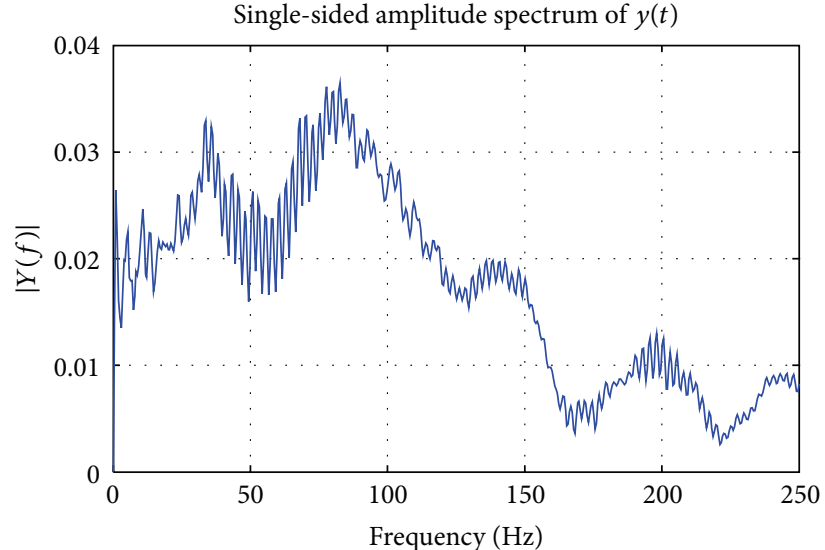

(b) Sensor 2 spectrum

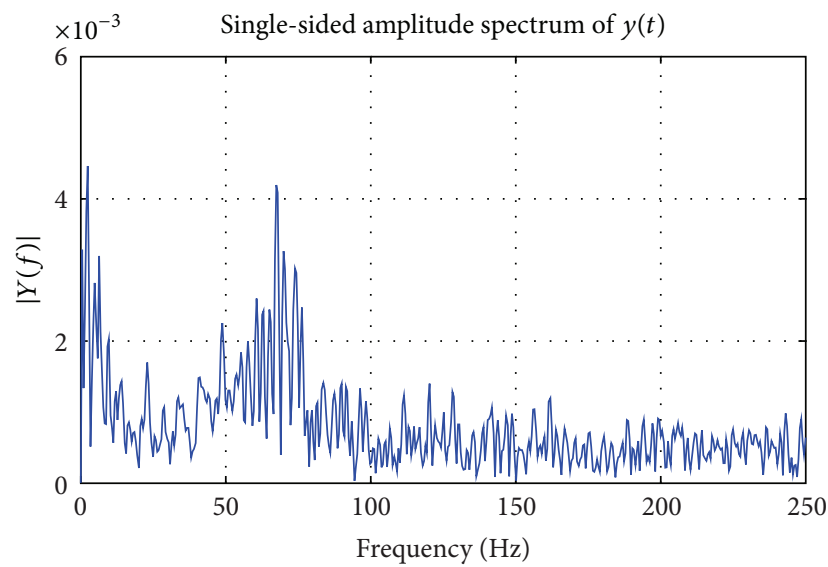

(d) Sensor 4 spectrum

FIGURE 10: Sensors spectrum of the landing mechanism with $V_{y}=0.75 \mathrm{~m} / \mathrm{s}, V_{x}=0 \mathrm{~m} / \mathrm{s}$, and $\theta=10^{\circ}$.

TABLE 4: Statistic analyses of natural frequency and maximum accelerations.

\begin{tabular}{|c|c|c|c|c|c|}
\hline & \multicolumn{2}{|c|}{ Natural frequency $(\mathrm{Hz})$} & \multicolumn{3}{|c|}{ Maximum acceleration (g) } \\
\hline & Modal analysis & Spectrum analysis & $V_{v}=0.41 \mathrm{~m} / \mathrm{s}$ & $V_{v}=0.75 \mathrm{~m} / \mathrm{s}$ & $V_{v}=1.17 \mathrm{~m} / \mathrm{s}$ \\
\hline Sensor 1 & $\begin{array}{c}37 \text { (mode } 7) \\
65(\text { mode } 9) \\
100(\text { mode } 15)\end{array}$ & 35,100 & 1.27 & 2.2 & 3.7 \\
\hline Sensor 2 & $\begin{array}{l}28(\text { mode } 6) \\
65(\text { mode } 9)\end{array}$ & 35,70 & 8.8 & 26.9 & 17.9 \\
\hline Sensor 3 & $\begin{array}{l}28(\text { mode } 6) \\
37(\text { mode } 7) \\
65(\text { mode } 9)\end{array}$ & 35 & 2.41 & 1.2 & 7.5 \\
\hline Sensor 4 & 65 (mode 9) & 70 & 0.47 & 0.78 & 1.64 \\
\hline
\end{tabular}

landing slope with foot 3 firstly, and then foot 2 and foot 1 collide with the landing slope almost simultaneously.

(iii) Maximum acceleration of each sensor increases with the landing velocity distinctly as shown in Table 4; besides the maximum acceleration of sensor 3 is $1.2 \mathrm{~g}$ when landing in the $V_{v}=0.75 \mathrm{~m} / \mathrm{s}$. This value is smaller than normal value. It may be induced by the direction of the acceleration sensor 3 which is not parallel to the landing direction because the pin connection between the landing leg and landing foot.

(iv) The largest acceleration is on foot 2 when landing and then is on foot 3 and foot 1 . The smallest acceleration is on equipment base. Accelerations of the equipment base in all landings are less than $10 \mathrm{~g}$. Thus, it can be found that the damping element plays an important role in protecting the instruments installed on equipment base. 


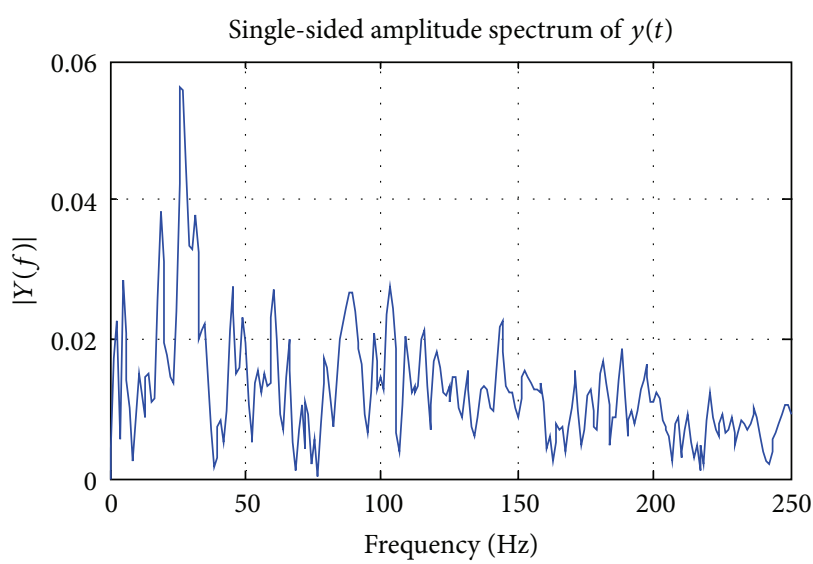

(a) Sensor 1 spectrum

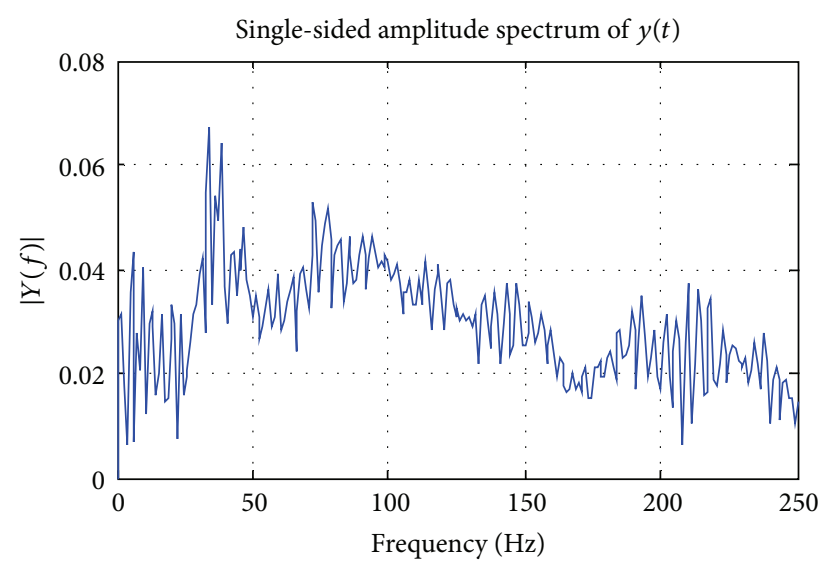

(c) Sensor 3 spectrum

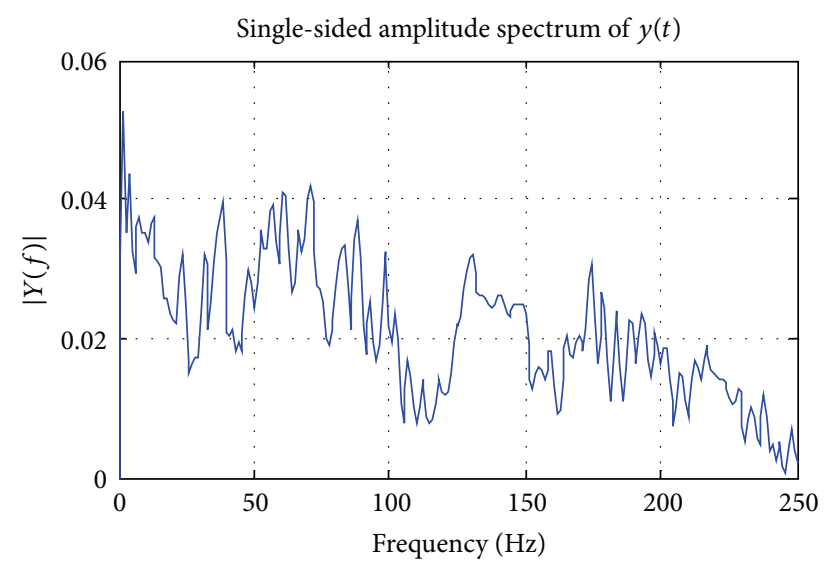

(b) Sensor 2 spectrum

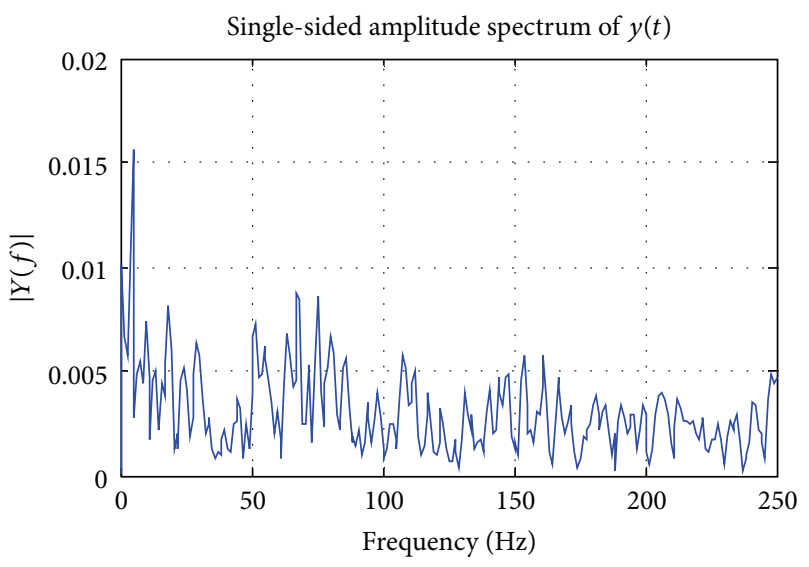

(d) Sensor 4 spectrum

FIGURE 11: Sensors spectrum of the landing mechanism with $V_{y}=1.17 \mathrm{~m} / \mathrm{s}, V_{x}=0 \mathrm{~m} / \mathrm{s}$, and $\theta=10^{\circ}$.

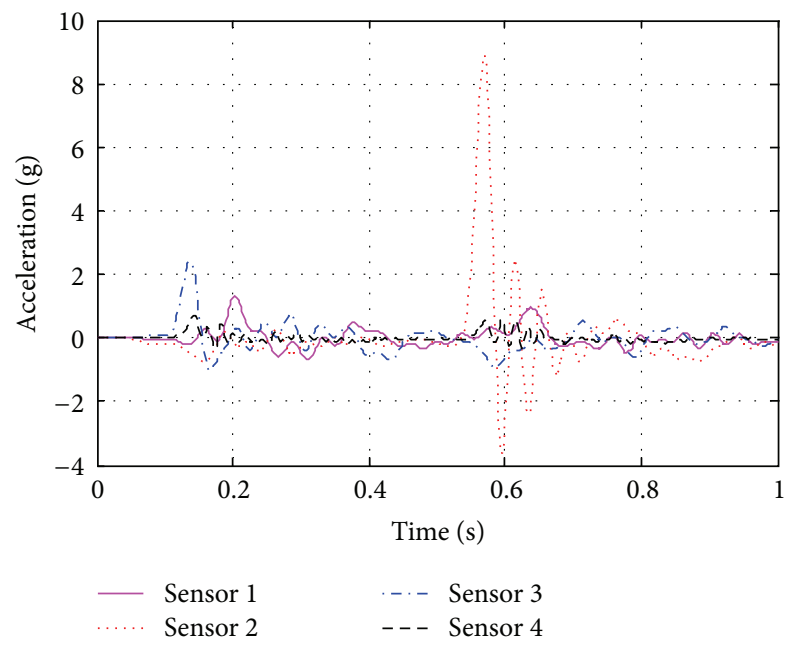

FIGURE 12: Accelerations with $V_{y}=0.41 \mathrm{~m} / \mathrm{s}, V_{x}=0 \mathrm{~m} / \mathrm{s}$, and $\theta=$ $10^{\circ}$.

The tilting of the landing mechanism can be measured by the gyroscope fixed on the landing mechanism. However, the gyroscope system is under developing. It will be equipped in future tests.

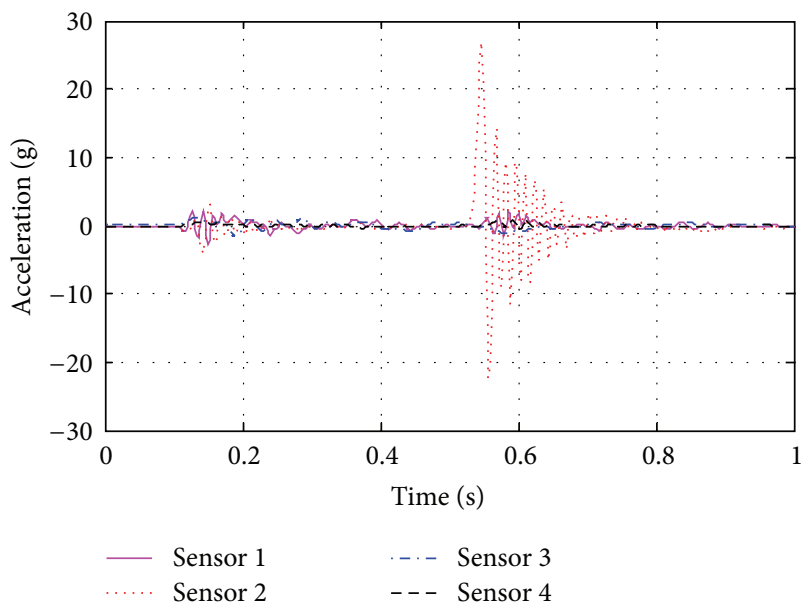

FIGURE 13: Accelerations with $V_{y}=0.75 \mathrm{~m} / \mathrm{s}, V_{x}=0 \mathrm{~m} / \mathrm{s}$, and $\theta=$ $10^{\circ}$.

\section{Conclusions}

ALISE landing mechanism to the asteroid with soft surface is developed. It has a mass of about $45 \mathrm{~kg}$, and the maximum 


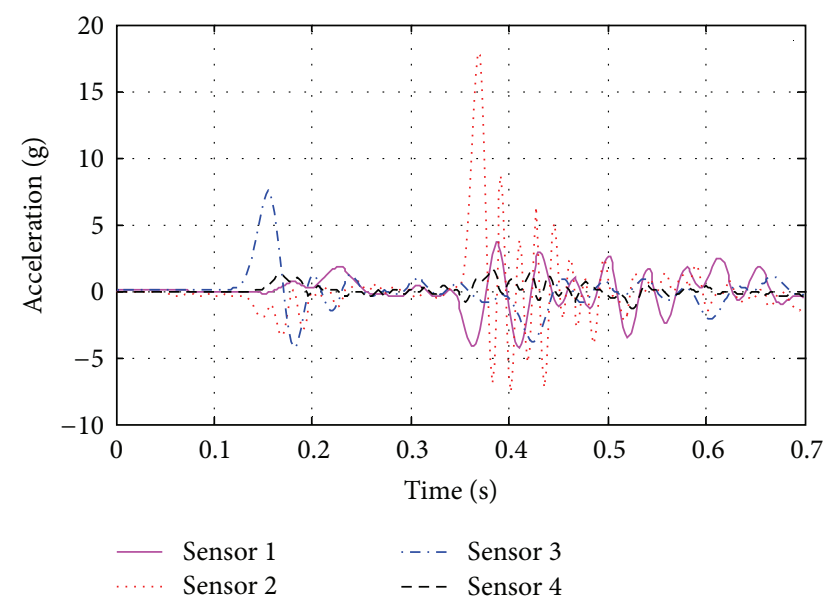

FIGURE 14: Accelerations with $V_{y}=1.17 \mathrm{~m} / \mathrm{s}, V_{x}=0 \mathrm{~m} / \mathrm{s}$, and $\theta=$ $10^{\circ}$.

payload is about $55 \mathrm{~kg}$. Its stiffness is high, and the modal frequency covers a wide range from $21.6 \mathrm{~Hz}$ to $100.7 \mathrm{~Hz}$, and the natural frequencies of the landing mechanism obtained by spectrum analyses of the landing accelerations are in accordance with the results of the FEA. The penetrating depth and anchoring force of the anchoring system are related to the cohesion of the medium. The larger the cohesion is, the smaller the penetrating depth is. Furthermore, the smaller the cohesion is, the steeper the penetrating depth changes with the penetrating velocity. Depth has less influence on the anchoring force in very low cohesion media. Landing impact of the landing mechanism is increased with the landing velocity, and the acceleration of the equipment base is less than $10 \mathrm{~g}$. Thus, the design of the landing mechanism is reasonable.

In the future, more penetrating and anchoring tests in various hard and soft media will be carried out to verify the performance of the anchoring system. At the same time, more landing with different attitude and velocity will be carried out on the air-floating platform to summarize the landing performance of the landing mechanism.

\section{Acknowledgments}

This work is financially supported by the National High Technology Research and Development Program of China (863 Program) (no. 2008AA12A214), the National Natural Science Foundation of China (no. 51105091), and the National Program on Key Research Program (no. 2013CB733103).

\section{References}

[1] R. P. Binzel, D. F. Lupishko, M. Di Martino et al., "Physical properties of near-earth objects," in Asteroids III, pp. 255-271, University of Arizona Press, Tucson, Ariz, USA, 2002.

[2] B. R. Blair, "The role of near-earth asteroids in long-term platinum supply," in Space Resources Roundtable II, pp. 1-15, University of Houston, Houston, Tex, USA, 2000.
[3] M. Yoshikawa and J. Kawaguchi, "Asteroid sample return mission Hayabusa, its engineering challenges and scientific results," in Proceedings of the 41st Lunar and Planetary Science Conference, March 2010.

[4] J. Veverka, M. Robinson, P. Thomas et al., "NEAR at Eros: imaging and spectral results," Science, vol. 289, no. 5487, pp. 2088-2097, 2000.

[5] J. Veverka, B. Farquhar, M. Robinson et al., "The landing of the NEAR-Shoemaker spacecraft on asteroid 433 Eros," Nature, vol. 413, no. 6854, pp. 390-393, 2001.

[6] J. Biele and S. Ulamec, "Capabilities of Philae, the Rosetta Lander," Space Science Reviews, vol. 138, pp. 275-289, 2008.

[7] M. D. Rayman and R. A. Mase, "Dawn's exploration of Vesta," Acta Astronautica, vol. 94, no. 1, pp. 159-167, 2013.

[8] H. Yano, T. Kubota, H. Miyamoto et al., "Touchdown of the Hayabusa spacecraft at the muses sea on Itokawa," Science, vol. 312, no. 5778, pp. 1350-1353, 2006.

[9] E. Asphaug et al., "Adventures in near-earth object exploration," Science, vol. 312, no. 5778, pp. 1328-1329, 2006.

[10] A. R. Ghavimi, F. Serricchio, F. Y. Hadaegh et al., "Autonomous landing and smart anchoring for in-situ exploration of small bodies," in Proceedings of the 5th International Symposium, pp. 1-6, ESTEC, Noordwijk, the Netherlands, 1999.

[11] Marshal Blessing, "Asteriods working group report," in Proceedings of the 2006 Next Generation Exploration Conference, August 2006.

[12] S. D. Ross, "Near-earth asteroid mining," Space Industry Report 107-81, Caltech, Pasadena, Calif, USA, 2001.

[13] T. J. Kelly, "Manned lunar lander design-the project Apollo Lunar module," in Proceedings of the AIAA Space Programs and Technologies Conference, pp. 1-10, Huntsville, Ala, USA, 1992.

[14] B. Donahue and C. R. Fowler, "Lunar lander configuration study and parametric performance analysis," pp. 1-19, AIAA/SAE/ASME/ASEE 29th Joint Propulsion Conference and Exhibit, Monterey, Calif, USA, 1993.

[15] S. Ulamec and J. Biele, "Surface elements and landing strategies for small bodies missions-philae and beyond," Advances in Space Research, vol. 44, no. 7, pp. 847-858, 2009.

[16] M. Hilchenbach, O. Küchemann, and H. Rosenbauer, "Impact on a comet: Rosetta Lander simulations," Planetary and Space Science, vol. 48, no. 5, pp. 361-369, 2000.

[17] A. D. Steltzner and A. K. Nasif, "Anchoring technology for in situ exploration of small bodies," in Proceedings of the 2000 IEEE Aerospace Conference, pp. 507-517, Big Sky, Mont, USA, July 2000.

[18] P. C. Thomas, J. Joseph, B. Carcich et al., "Eros: shape, topography, and slope processes," Icarus, vol. 155, no. 1, pp. 18-37, 2002.

[19] A. Fujiwara, J. Kawaguchi, D. K. Yeomans et al., "The rubble-pile asteroid Itokawe as observed by Hayabusa," Science, vol. 312, no. 5778, pp. 1330-1334, 2006.

[20] B. Schläppi, K. Altwegg, and P. Wurz, "Asteroid exosphere: a simulation for the ROSETTA flyby targets (2867) Steins and (21) Lutetia," Icarus, vol. 195, no. 2, pp. 674-685, 2008.

[21] P. L. Lamy, I. Toth, B. J. R. Davidsson et al., "A portrait of the nucleus of comet 67P/Churyumov-Gerasimenko," Space Science Reviews, vol. 128, pp. 23-66, 2007.

[22] P. L. Lamy, I. Toth, L. Jorda, H. A. Weaver, and M. A'Hearn, “The nucleus and inner coma of comet 46P/Wirtanen," Astronomy and Astrophysics, vol. 335, no. 2, pp. L25-L29, 1998. 

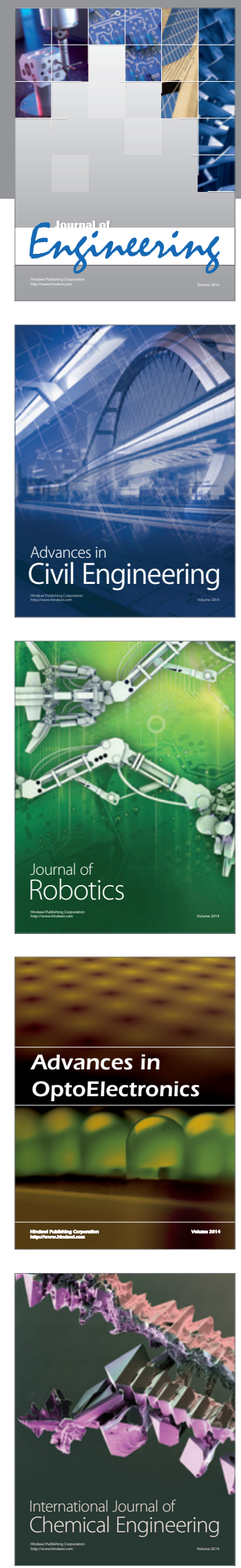

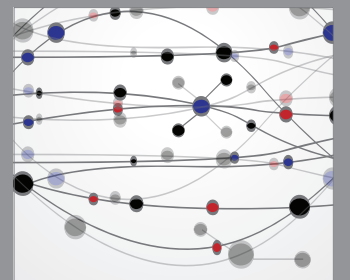

The Scientific World Journal
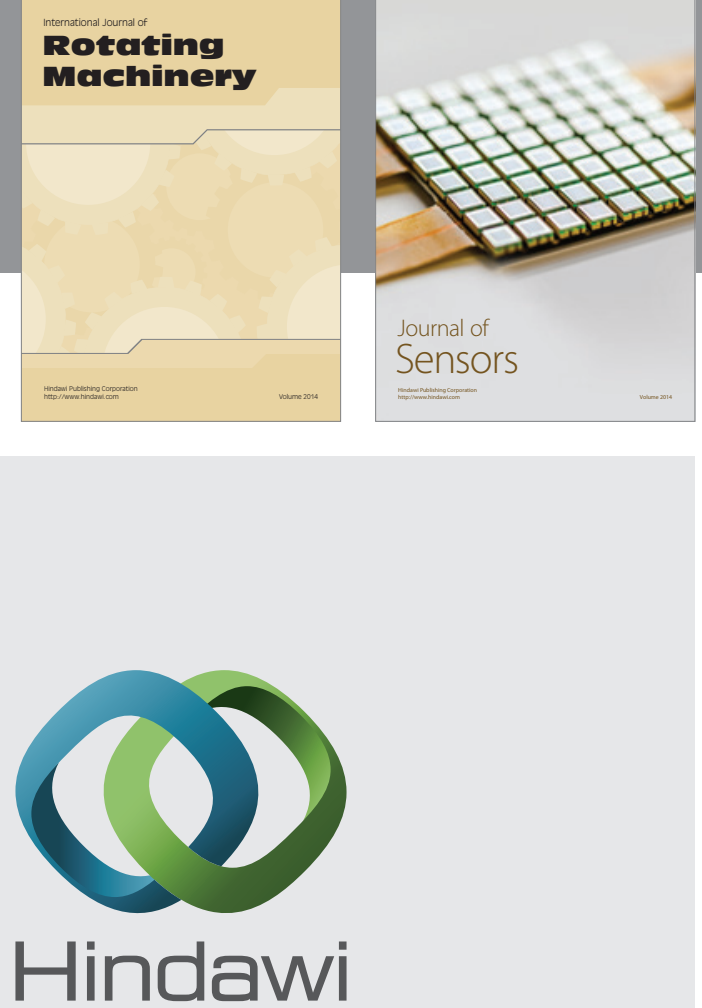

Submit your manuscripts at http://www.hindawi.com
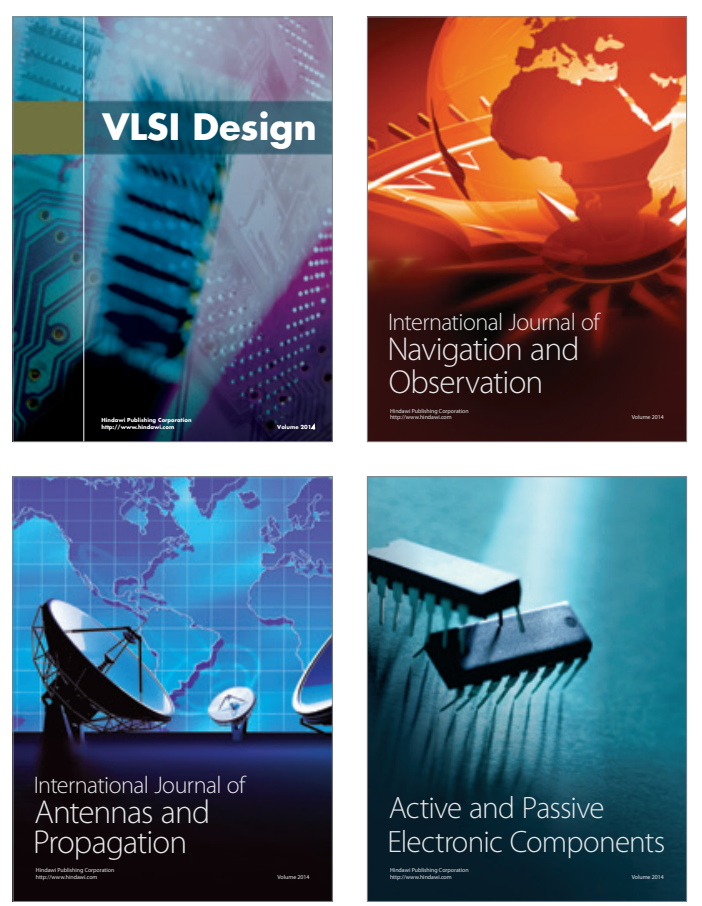
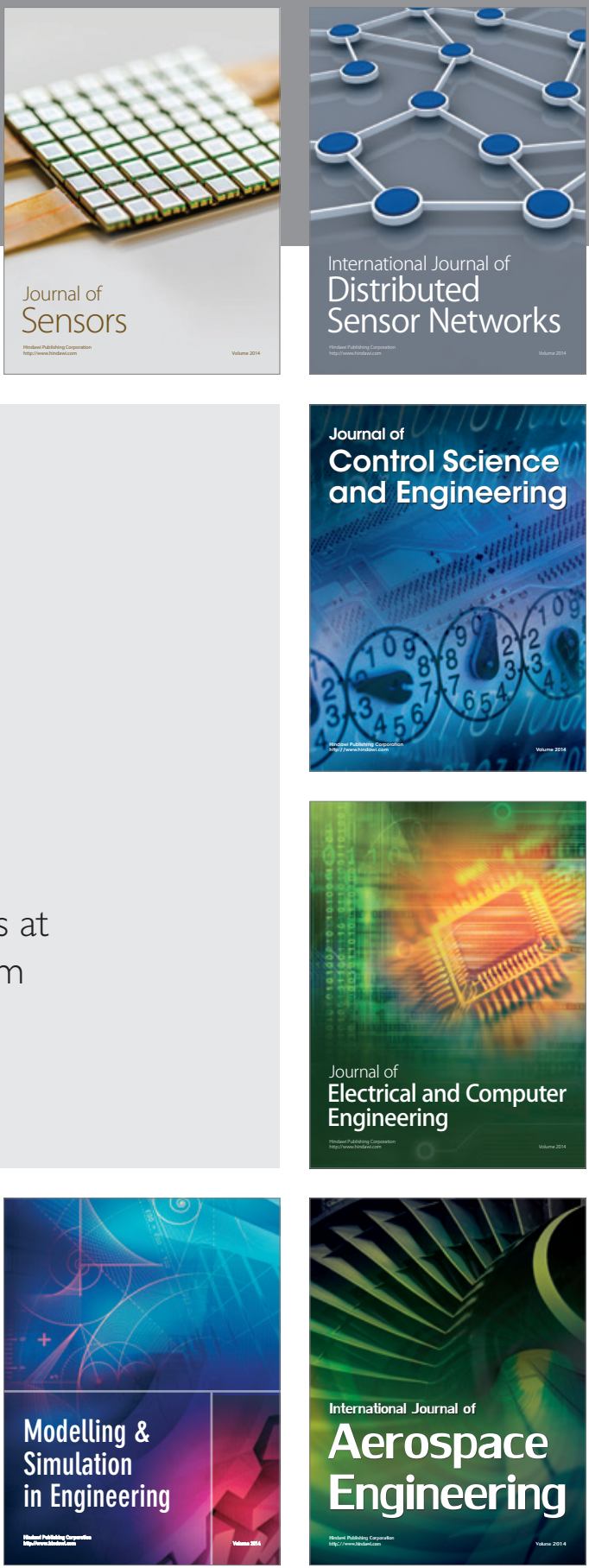

Journal of

Control Science

and Engineering
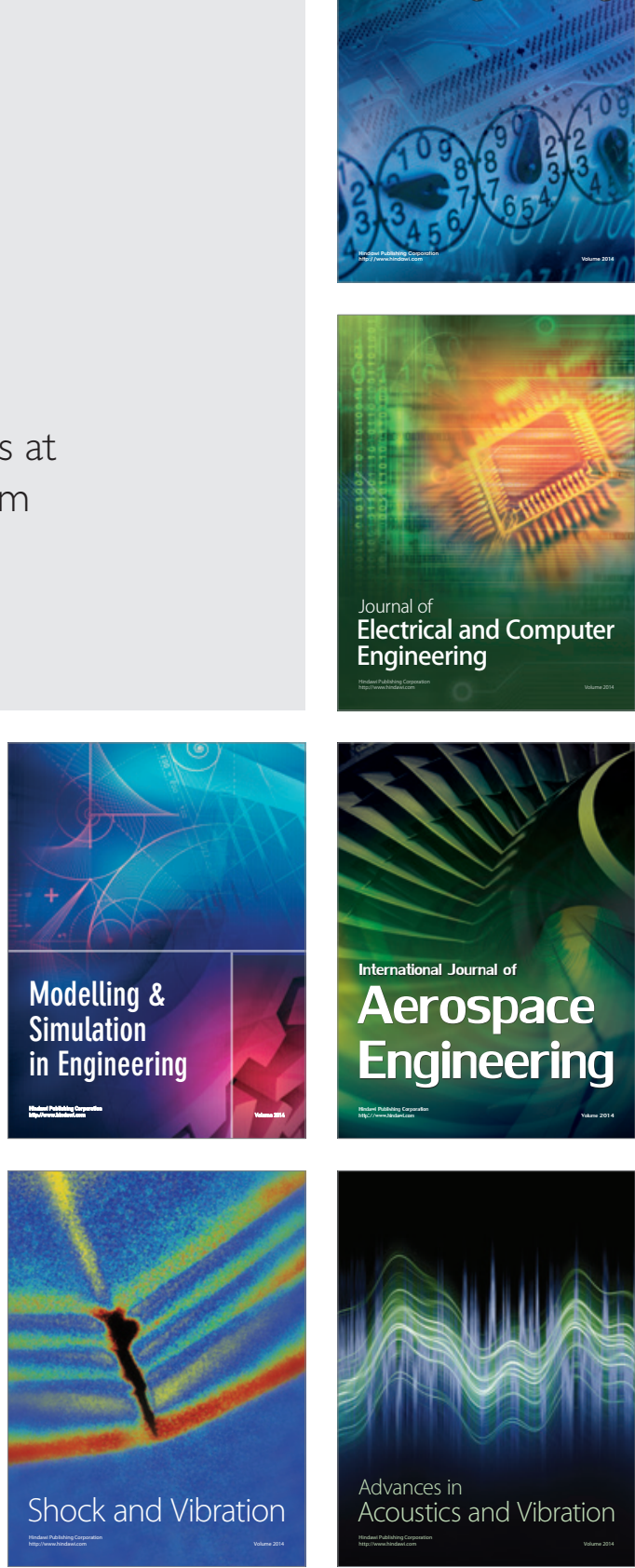